\title{
Adaptation of POS Tagging for Multiple BioMedical Domains
}

\author{
John E. Miller ${ }^{1}$ \\ ${ }^{1}$ Computer \& Information Sciences \\ University of Delaware \\ Newark, DE 19716 \\ \{jmiller,vijay\}@cis.udel.edu
}

\author{
Manabu Torii ${ }^{2}$ \\ K. Vijay-Shanker ${ }^{1}$ \\ ${ }^{2}$ Biostatistics, Bioinformatics and Biomathematics \\ Georgetown University Medical Center \\ Washington, DC 20057 \\ mt352@georgetown.edu
}

\section{Introduction}

Part of Speech (POS) tagging is often a prerequisite for tasks such as partial parsing and information extraction. However, when a POS tagger is simply ported to another domain the tagger's accuracy drops. This problem can be addressed through hand annotation of a corpus in the new domain and supervised training of a new tagger. In our methodology, we use existing raw text and a generic POS annotated corpus to develop taggers for new domains without hand annotation or supervised training. We focus in particular on out-ofvocabulary words since they reduce accuracy (Lease and Charniak. 2005; Smith et al. 2005).

There is substantial information in the derivational suffixes and few inflectional suffixes of English. We look at individual words and their suffixes along with the morphologically related words to build a domain specific lexicon containing POS tags and probabilities for each word.

\section{Adaptation Methodology}

Our methodology is described in detail in Miller et al (2007) and summarized here: 1) Process generic POS annotated text to obtain state and lexical POS tag probabilities. 2) Obtain a frequency table of words from a large corpus of raw sub-domain text. 3) Construct a partial sub-domain lexicon matching relative frequencies of morphologically related words with words from the generic annotated text averaging POS probabilities of the $\mathrm{k}$ nearest neighbors. 4) Combine common generic words and orthographic word categories with the partial lexicon making the sub-domain lexicon. 5) Train a first order Hidden Markov Model (HMM) by Expectation Maximization (EM). 6) Apply the Viterbi algorithm with the HMM to tag subdomain text.

\section{Adaptation to Multiple Domains}

Molecular Biology Domain: We used the Wall Street Journal corpus (WSJ) (Marcus et al, 1993) as our generic POS annotated corpus. For our raw un-annotated text we used 133,666 abstracts from the MEDLINE distribution covering molecular biology and biomedicine sub-domains. We split the GENIA database (Tateisi et al, 2003) into training and test portions and ignored the POS tags for training. We ran a 5 -fold cross validation study and obtained an average accuracy of $95.77 \%$.

Medical Domain: Again we used the WSJ as our generic POS annotated corpus. For our raw unannotated text we used 164,670 abstracts from the MEDLINE distribution with selection based on 83 journals from the medical domain. For our HMM EM training we selected 1966 abstracts (same journals). For evaluation purposes, we selected 1932 POS annotated sentences from the MedPost (Smith et al, 2004) distribution (same journals). The MedPost tag set coding was converted to the Penn Treebank tag set using the utilities provided with the MedPost tagger distribution. We obtained an accuracy of $93.17 \%$ on the single medical test corpus, a substantial drop from the $95.77 \%$ average accuracy obtained in the GENIA corpus.

\section{Coding Differences}

We looked at high frequency tagging errors in the medical test set and found that many errors resulted directly from the differences in the coding styles between GENIA and MedPost. Our model reflects the coding style of the WSJ, used for our generic POS annotated text. GENIA largely followed the WSJ coding conventions. Annotation in the 1932 sentences taken from MedPost had some systematic differences in coding style from this. 
Identified Differences: Lexical differences: 1) Words such as 'more' and 'less' are JJR or RBR in WSJ/GENIA but JJ or RB in MedPost. 2) Tokens such as $\%,=, l,<,>$ are typically $\mathrm{NN}$ or $\mathrm{JJ}$ in WSJ/GENIA but SYM in MedPost. 3)'be' is VB in WSJ/GENIA but VB or VBP in MedPost. 4) Some orthographic categories are $\mathrm{JJ}$ in WSJ/GENIA but NN in MedPost. Transition discrepancies: 1) Verbs are tagged VB following a TO or MD in WSJ/GENIA but only following a TO in MedPost. 2) MedPost prefers NN and NN-NN sequences.

Ad Hoc Adjustments: We constructed a new lexicon accounting for some of the lexical differences and attained an accuracy of $94.15 \%$ versus the previous $93.17 \%$. Next we biased a few initial state transition probabilities, changing $\mathrm{P}(\mathrm{VB} \mid \mathrm{MD})$ from very high to a very low and increasing $\mathrm{P}(\mathrm{NN} \mid \mathrm{NN})$, and attained an accuracy of $94.63 \%$.

As the coding differences had nothing to do with suffixes and suffix distributions, the central part of our methodology, we tried some ad hoc fixes to determine what our performance might have been. We suffered at least a $1.46 \%$ drop in accuracy due to differences in coding, not language use.

\section{Evaluation}

The table shows the accuracy of our tagger and a few well-known taggers in our target biomedical sub-domains.

\begin{tabular}{|l|l|}
\hline Molecular Biology & \%Accuracy \\
\hline - Our tagger (5-fold) & $95.8 \%$ \\
\hline - MedPost & $94.1 \%$ \\
\hline - Penn BioIE & $95.1 \%$ \\
\hline - GENIA supervised & $98.3 \%$ \\
\hline Medical Domain & \\
\hline - Our tagger & $93.17 \%$ \\
\hline - Our tagger (+ lex bias) & $94.15 \%$ \\
\hline - Our tagger (+ lex \& trans bias) $^{1}$ & $94.63 \%$ \\
\hline - MedPost supervised $^{2}$ & $96.9 \%$ \\
\hline
\end{tabular}

The MedPost and Penn BiolE taggers used annotated text and supervised training in other biomedical domains, but they were not trained specifically for the GENIA Molecular Biology subdomain. Our tagger seems competitive with these

\footnotetext{
${ }^{1}$ PennBioIE. 2005. Mining The Bibliome Project. http://bioie.ldc.upenn.edu/.

${ }^{2}$ Based on Medpost test set of 1000 sentences, not on our test set of 1932 sentences.
}

taggers. We cannot claim superior accuracy as these taggers may suffer the same coding bias effects we have noted. The superior performance of the GENIA tagger (Tsuruoka et al. 2005) in the Molecular Biology/GENIA domain and the MedPost tagger (Smith et al. 2004) in its biomedical domain owes to their use of supervised training on an annotated training set with evaluation on a test set from the same domain. The approximate 1.5\% bias effect due to coding differences is attributable to organizational differences in POS.

\section{Conclusions}

To cope with domain specific vocabulary and uses of vocabulary, we exploited the suffix information of words and related words to build domain specific lexicons. We trained our HMM using EM and un-annotated text from the specialized domains. We assessed accuracy versus annotated test sets in the specialized domains, noting discrepancies in our results across specialized domains, and concluding that our methodology performs competitively versus well-known taggers that used annotated text and supervised training in other biomedical domains.

\section{References}

M. Lease and E. Charniak. 2005. Parsing Biomedical Literature. IJCNLP-05: 58-69.

M. Marcus, B. Santorini, M.A. Marcinkiewicz. 1993. Building a large annotated corpus of English: The Penn Treebank. Comp. Ling., 19:313-330.

J.E. Miller, M. Torii, K. Vijay-Shanker. 2007. Building Domain-Specific Taggers Without Annotated (Domain) Data. EMNLP-07.

L. Smith, T. Rindflesch, W.J. Wilbur. 2004. MedPost: a part-of-speech tagger for bioMedical text. Bioinformatics 20 (14):2320-2321.

L. Smith, T. Rindflesch, W.J. Wilbur. 2005. The importance of the lexicon in tagging biomedical text. Natural Language Engineering 12(2) 1-17.

Y. Tateisi, T. Ohta, J. Dong Kim, H. Hong, S. Jian, J. Tsujii. 2003. The GENIA corpus: Medline abstracts annotated with linguistic information. Third meeting of SIG on Text Mining, ISMB.

Y. Tsuruoka, Y. Tateishi, J.D. Kim, T. Ohta, J. McNaught, S. Ananiadou, J. Tsujii. 2005. Developing a Robust Part-of-Speech Tagger for Biomedical Text, Advances in Informatics, LNCS 3746: 382-392. 